\title{
Der Mumiendoktor
}

\section{Erhard Taverna}

Die Mumienforschung ist ein weltweit vernetztes Forschungsgebiet, in dem die Universität Zürich mit ihrem «Swiss Mummy Project» eine führende Rolle spielt. Das neue Spezialgebiet, häufig als Paläopathologie oder Paläoradiologie bezeichnet, ist eine Wissenschaft, die mit modernsten medi-

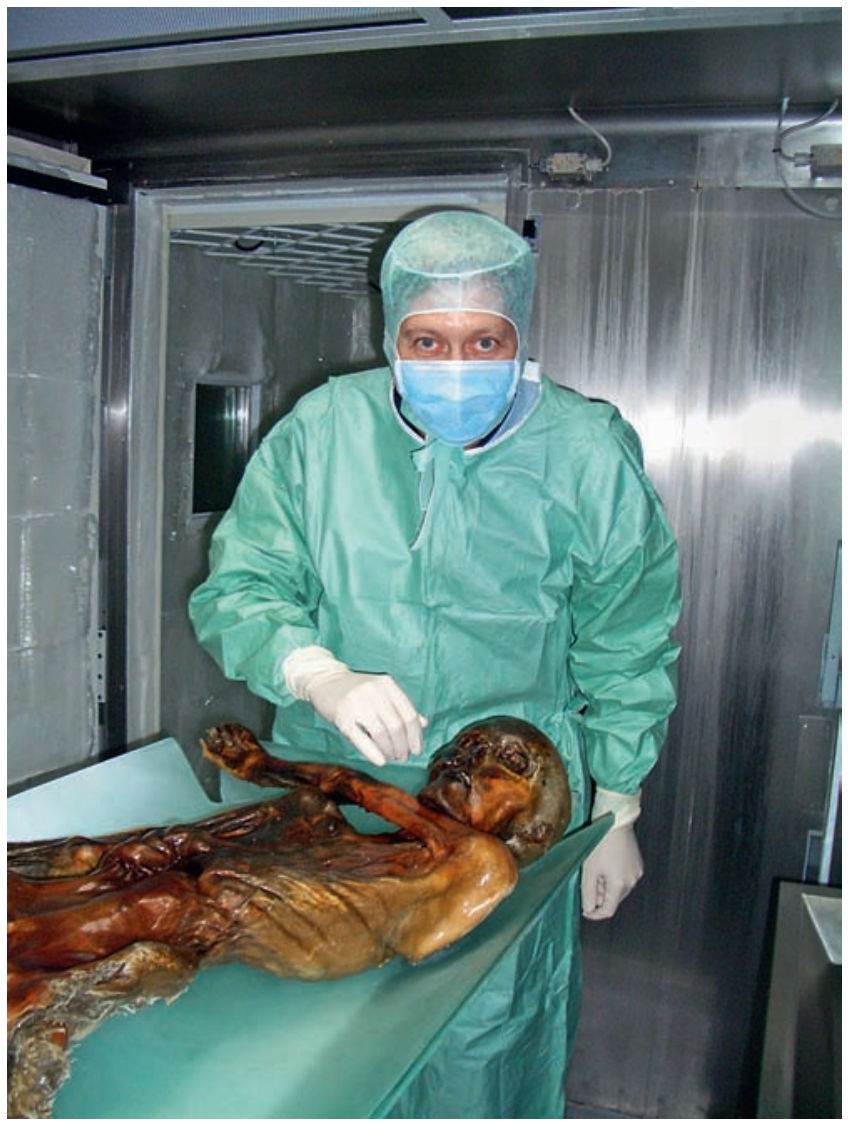

* PD Dr. Dr. Frank J. Rühli, Anatomisches Institut der Universität Zürich Winterthurerstrasse 190 8057 Zürich, E-Mail: frank.ruhli@anatom.uzh.ch

1 Rühli FJ, Henneberg M, Schär DJ, Imhof A, Schleiffenbaum B, Woitek U. Determinants of interindividual cholesterol levels variations in an unbiased young male sample Swiss Med Wkly. 2008;138(19-20):286-91. anatomischen Kenntnissen Anthropologie und Forensik um eine weitere Dimension bereichert.

Frank J. Rühli*, geboren 1971, gehört zu den Stars der Disziplin. Wenig im nüchternen Büro des anatomischen Institutes der Universität Zürich verrät seinen Beruf, nur das kanadische Poster mit dem Titel «Palaeoradiology of mummies, skeletal remains and hominid fossils» und die Figurengruppe mit dem Einbalsamierer auf dem Schreibtisch. Schon als Kind habe er sich für das Thema interessiert und während des Medizinstudiums über altägyptische Mumien dissertiert. Weitere Etappen führten über die Radiologie zu zinisch-diagnostischen Methoden und exakten einem PhD in Australien und über zahlreiche wissenschaftliche Veröffentlichungen und Lehrverpflichtungen zur Antrittsvorlesung im Herbst, wie es sich für einen Privatdozenten gehört. Wenn Frank Rühli nicht gerade publiziert oder Fundstätten und Kongresse besucht, begleitet er Doktoranden, hält Anatomievorlesungen, leitet Präparier- und Histologiekurse, examiniert an Prüfungen und sucht nach Geldgebern. Die Beschäftigung mit prominenten Toten wie dem Eis-mann im Südtirol oder dem Pharao Tutanchamun sichern ihm die Aufmerksamkeit der Medien und das Interesse unentbehrlicher Sponsoren und Kollaborateure wie des Schweizerischen Natinalfonds oder der Firma Siemens. Denn mit weiterentwickelten MRI-Technologien ist es möglich, Mumien an Ort und Stelle mit nichtinvasiven Methoden zu untersuchen. Obwohl mumifiziertes Gewebe kaum Wasser enthält, werden mit dem NMR-Mouse (Mobile Universal Surface Explorer $^{\odot}$ ) dünne Schichten wie Fingernägel oder Bandagen sichtbar. Millimetergenaue Vergleichsprofile an Modellen und Freiwilligen ermöglichen, feinste anatomische Details zu erkennen. 2005 wurde die Gletschermumie von Ötzi (3300 v. Chr.) im Kühlraum des Archäologiemuseums in Bozen untersucht und ebenso im Spital mittels CT als Todesursache eine Blutung aus der linken Arteria subclavia nachgewiesen. Das neolithische Corpus delicti liegt als steinerne Pfeilspitze, wie CT-basierte 3-D-Rekonstruktionen eindeutig belegen, ventral der Scapula. Bei solchen Untersuchungen sind oft Teile von Bandscheiben und degenerative Veränderungen, Arteriosklerose oder gar einzelne Komponenten der Einbalsamierungssubstanz analysierbar. Tutanchamun wurde im Tal der Könige mit einem mobilen CT-Gerät untersucht, wobei eine bisher unbekannte distale Femurfraktur entdeckt wurde. Erfolgte sie zu Lebzeiten oder postmortal? Vielleicht werden histologische Untersuchungen den Fall klären, so wie DNS-Proben im von Frank Rühli geplanten Labor weitere Krankheiten und dynastische Querverbindungen aufklären sollen.

Frank Rühli interessieren die Langzeitverläufe der menschlichen Pathologie. Die Veränderungen an mittelalterlichen Wirbelsäulen, degenerative, metabolische, tumoröse und infektiöse Befunde von Männern und Frauen, die ausser der 
medizinhistorischen auch eine aktuelle Bedeutung haben. Eine Knochensammlung der Pathologie aus den 50er und 60er Jahren ist trotz Geldmangel im Aufbau und wird mit den zugehörigen Patientendaten vergleichende Langzeitstudien ermöglichen. Auch historisch näher liegende Krankheiten können überraschende Aktualität erlangen, wie - durch eine andere Arbeitsgruppe die Exhumierung gefrorener Leichen in Island belegte, die noch Viren der Spanischen Grippe enthielten. Doch die epidemiologische Neugier von Frank Rühli und seinen Arbeitsgruppen reicht bis in die Gegenwart, wie eine neue Studie im «Swiss Medical Weekly» zum Cholesterinspiegel von Stellungspflichtigen und dessen Risikofaktoren zeigt [1].

Eine mehrtausendjährige Arterienverkalkung verbindet die Menschen von den Pyramiden mit den ethno-kulturellen Besonderheiten junger Schweizer Männer. Das wird nicht die letzte Erkenntnis der Mumienforscher bleiben, die mit allen technischen Tricks die Verstorbenen zum

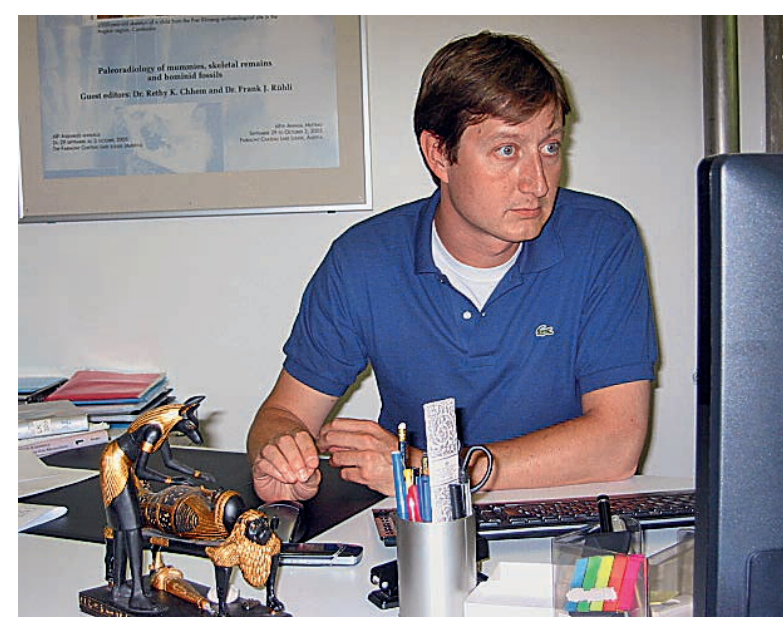

Sprechen bringen. Ihre Patienten bleiben mausetot, und die Forscher haben nicht zu befürchten, dass eine Mumie wieder zum Leben erwacht, wie das Boris Karloff im Gruselklassiker von 1932 unvergesslich vorführte.

\section{Himmelsweg}

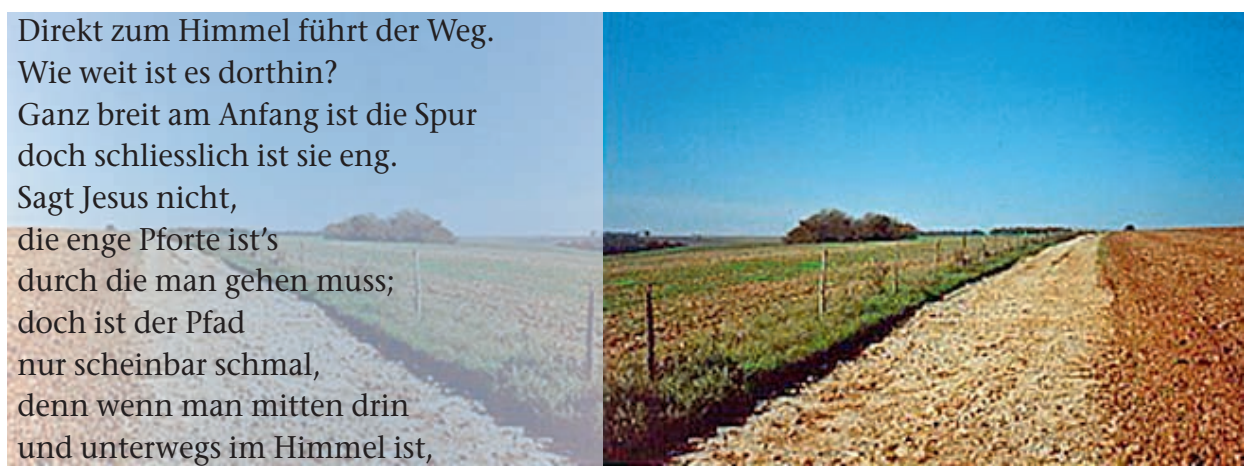

dann plötzlich wird es weit und gross.

Der Himmel ist unendlich.

David Künzler, Hausen am Albis 